\title{
Numerical and experimental evaluation of performance of centrifugal seals
}

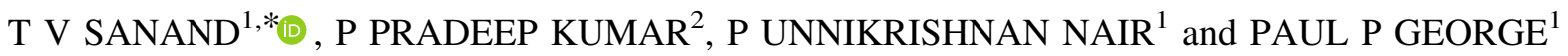 \\ ${ }^{1}$ Liquid Propulsion System Centre, Indian Space Research Organisation, \\ Valiamala, Thiruvananthapuram 695547, India \\ ${ }^{2}$ Department of Aerospace Engineering, Indian Institute of Space Science and Technology, \\ Valiamala, Thiruvananthapuram 695547, India \\ e-mail: sanandtv@gmail.com
}

MS received 30 April 2016; revised 12 September 2016; accepted 4 October 2016

\begin{abstract}
Centrifugal seals' or 'Slinger seals' offer an attractive choice as non-contact-type sealing in fluid machinery. These seals utilize the radial pressure gradient caused by centrifugal forces in a rotating fluid ring, to create a sealing of the working fluid. Basic construction of a typical seal consists of a rotating disc inside a stationary casing; one side of the disc (sealing side) is provided with a set of slots (Type-1) or vanes (Type-2) to enhance the tangential velocity of the fluid. The other side of the disk (back side) in both the configurations is exposed to high pressure liquid being sealed. Both numerical and experimental investigations of the performance of Type-1 seal (with slots) have been carried out so as to optimize the seal configuration to achieve maximum sealing capacity, with minimum power consumption. A comparison of the performance of Type-1 seal has been made with that of conventional one (Type-2) in view of economy of construction and better sealing with minimal expense of power consumption. A test rig that allows for varying the major geometrical and operating parameters was designed and tests were conducted with water as the medium. Influence of major geometric parameters like dimensions and number of slots, axial/radial clearances and major operating parameters like rotational speed, inlet pressure and sealing fluid bypass flow rate has been investigated. Apart from various pressure, temperature, flow and torque measurements, the interface between the sealing and working fluid for the experiments was captured and recorded using a high speed camera at $\sim 26,000$ frames per second. Geometrical configuration for the slots that maximizes the sealing capacity is arrived through 3D numerical simulations using commercial CFD solver ANSYS Fluent ${ }^{\circledR}$. A good agreement is obtained with respect to experimental results. In view of economy of construction and better sealing with minimal expense of operating power, a modified version of Type-1 seal termed as Type-3 seal is investigated. A simple 1D model for prediction of the interface radius during the seal operation, which could be used as a quick design guide, is also presented.
\end{abstract}

Keywords. Centrifugal seals; slinger seals; sealing impellers.

\section{Introduction}

A good overview of sealing technology is provided in [1]. Centrifugal seals form a class of dynamic non-contact-type seals that have a potential to ensure complete leak tightness during operation, unlike other conventional non-contacttype seals like labyrinth seals and floating ring seals. A typical centrifugal seal configuration is shown in figure 1 . The back side of the seal is exposed to the high pressure liquid being sealed. A small pressure rise occurs (radially outwards) across the back side of the disk due to the pumping action of the rotating disc. On the other side, there is a much higher pressure drop (radially inwards) across the vaned/slotted side by virtue of the higher angular velocity

*For correspondence imparted to the fluid by the vanes/slots. Eventually, a liquid-air interface is formed, where the pressure becomes equal to that of outside ambient air and sealing is achieved.

The work by Theodore Von Karman in 1921 [2] is one of the earliest fundamental references in analysing the flow over a free rotating disc. His theoretical investigations on the laminar and turbulent flow created by a rotating disk are detailed in [2]. He derived a solution of the Navier-Stokes equations using momentum integral approach, assuming a steady, axi-symmetric laminar flow over a rotating disc of infinite radius situated in a quiescent viscous fluid, which extends up to infinity. He derived an expression for the torque required to maintain the speed of the disc in terms of a moment coefficient $C_{\mathrm{m}}$. The same theory was extended for the turbulent case also, through momentum integral approach by assuming a $1 / 7$ th power law for velocity 


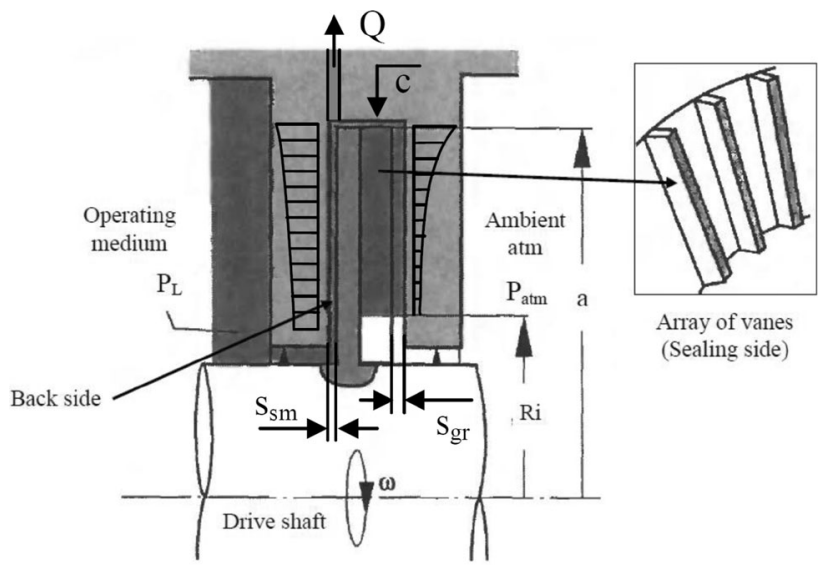

Figure 1. A typical rotating disc centrifugal seal [1].

distributions and semi-empirical expressions for shear stresses in the vicinity of the disc. Subsequent works by Cochran [3] and Goldstein [4] provided improvements to his analysis. Daily and Nece [5] proposed four possible flow regimes inside a stator-rotor cavity in terms of rotational Reynolds number $\operatorname{Re}$ and $s / a$ ratio as follows (figure 2):

\section{I laminar merged boundary layer flow,}

II laminar separated boundary layer flow,

III turbulent merged boundary layer flow and

IV turbulent separated boundary layer flow.

Due to the rotation of the disc and retardation of the stationary wall, boundary layers with radial velocities are formed in the vicinity of the respective walls. Depending on the high enough value of the axial gap ' $s$ ' between the walls, there exists an inviscid fluid core under solid body rotation, which excludes any radial velocities. The ratio of tangential velocity of the fluid inside the core to that of the rotor, known as core rotation factor ' $K$ ', is the single most important parameter defining the radial pressure rise. Here, the balance of moments due to rotating and stationary elements was used to obtain $K$. From the balance of radial pressure rise and centrifugal forces, the radial pressure gradient can be expressed in the following form [5]:

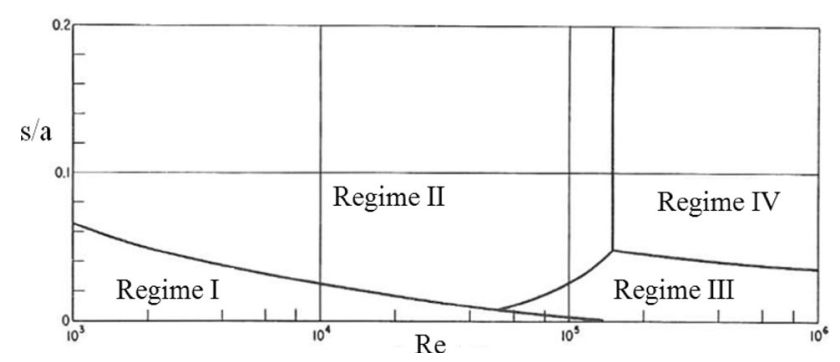

Figure 2. Flow regimes for an enclosed rotating disc [5].

$$
\frac{\partial p}{\partial r}=\rho(K \omega)^{2} r
$$

They provided expressions for estimating the moment coefficients as a function of $\mathrm{Re}$ and $s / a$. The same methodology was later extended by Ketola and McGrew [6], for the case of a partially wetted rotating disc inside a confinement, which is the basic analytical model for a simple plain disc centrifugal seal except for the presence of vanes/slots. They introduced a new term called submergence ratio $X$, which is a measure of the degree of submergence of the disc in liquid. The effect of a radially outward flow rate on core rotation factor of a stator-rotor cavity was later modelled by Owen [7], according to whom the core rotation factor was found to vary with respect to the flow rate, radial location along the disc and the Reynolds number. He also provided expressions for moment coefficient $C_{\mathrm{m}}$ as a function of the above parameters.

Arndt [8] details his experimental studies on enclosed rotating disc superposed with throughflows are. His focus was on the effect of a superimposed radially outward throughflow on the radial pressure distribution inside a stator-rotor cavity. Expressions for $K$ and $C_{\mathrm{m}}$ were derived from simple theoretical analyses that contained unknown coefficients to be determined from a large number of tests. The effect of radial ribs attached to the disc surface on the core rotation factor inside a stator-rotor cavity was investigated by Due Jr [9]. He provided empirical expressions for estimating core rotation factor as a function of various geometrical and operating parameters for the discs. The performance study of a centrifugal seal by monitoring the liquid interface with the help of a high speed camera and torque measurements using a torque meter was done by Marcu et al [10]. The experimental set-up for the current study is developed based on this work.

Among numerical studies, the case of a stator-rotor cavity superposed with a radially outward flow rate was investigated by Poncet [11]. He related the core rotation factor to the superposed flow rate using a non-dimensional flow rate parameter $\mathrm{Cq}_{\mathrm{r}}$, combining the effects of flow rate, angular velocity and radial location of the point under consideration. He provided two empirical correlations for estimating $K$ as a function of $\mathrm{Cq}_{\mathrm{r}}$. The usage of commercial CFD solvers for simulations of stator-rotor cavities of typical configurations to determine the core rotation factor $K$ was demonstrated by Poncet et al [12] in their work. Prediction of moment coefficient $C_{\mathrm{m}}$ for an enclosed rotating disc through numerical simulations using commercial CFD solvers is carried out by Beretta and Malfa [13].

\section{Methodology}

The influence of the sealing side geometry on the sealing capacity of the seal, i.e., the pressure difference that can be applied across the seal during the operation, is studied 
numerically by carrying out 3D simulations on various geometrical combinations for the slots (Type-1 seal). Based on the results of the simulations, selected seal geometries were manufactured and tests were carried out. A good agreement was found between the numerical simulations and test results. Both Type- 1 and Type- 2 seals are experimentally characterized. It has been possible to systematically arrive at an improved version of Type-1 seal configuration; the improved design is designated as Type-3.

\subsection{Numerical studies (sealing side geometry)}

The parameters and their range of investigation are given in table 1 . In a particular analysis only one parameter was varied to study its influence. Selection of these geometrical parameters was in line with the work done by Due Jr [9].

The core rotation factor for the slotted/grooved side of the seal $\left(K_{\mathrm{gr}}\right)$ is a direct measure of its sealing capacity, which can be readily observed from (1). The changes in $K_{\mathrm{gr}}$ with respect to changes in the geometrical parameters are investigated in these simulations. The combination of geometries that maximizes $K_{\mathrm{gr}}$ and hence sealing capacity is the best one for the sealing side.

The computational domain under consideration is shown in figure 3. A $1 / n$ sector $(n=6-24)$ of the full $360^{\circ}$ domain is modelled using structured grids. Grid independence is confirmed by performing simulations on three successive grid refinements with cell sizes 17,82,974 (Mesh-1), 34,36,724 (Mesh-2) and 45,20,299 (Mesh-3). The ratio of tangential velocity of the fluid to that of the disc was

Table 1. Geometrical parameters studied for assessment of effect of slots/grooves on seal performance.

\begin{tabular}{lccc}
\hline$\#$ & Parameter & Legend & Range \\
\hline 1. & Number of slots & $n$ & $6-24$ \\
2. & Width of slots & $w$ & $1-5 \mathrm{~mm}$ \\
3. & Depth of slots & $h$ & $1-5 \mathrm{~mm}$ \\
\hline
\end{tabular}

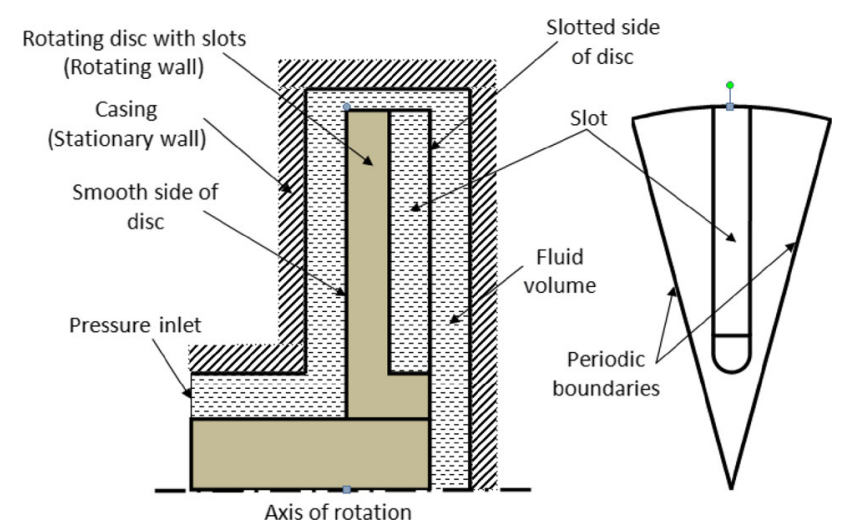

Figure 3. Axi-symmetric computational domain considered for parametric study of slots.

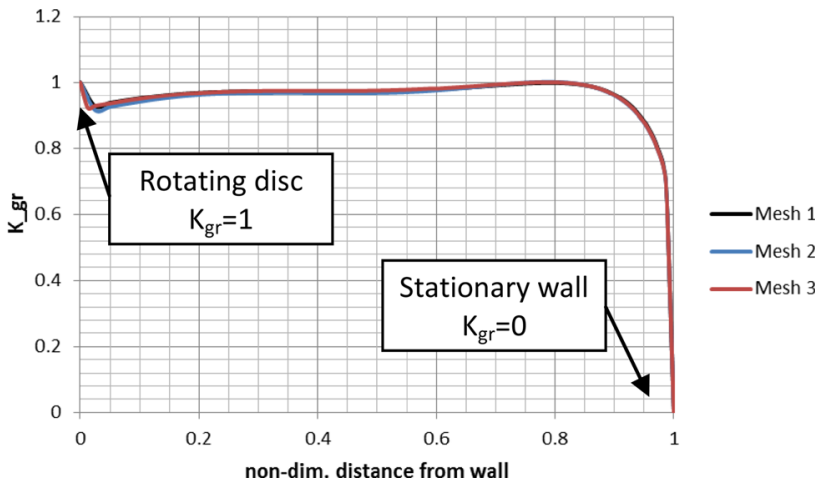

Figure 4. Comparison of $K_{\mathrm{gr}}$ factor on slotted side for Mesh 1-3.

compared at various radial and axial locations along the disc. A comparison of the core rotation factor for the slotted side of the seal $\left(K_{\mathrm{gr}}\right)$ for the afore-mentioned three cases is shown in figure 4 , wherein a very close match was observed among the three. Mesh-1 was selected for further simulations as a compromise between accuracy and computational cost. The standard $k-\varepsilon$ model with scalable wall function was used as the turbulence model as full resolution of the boundary layer resulted in enormous increase in the grid sizes and hence computational effort. Near-wall meshing was done to ensure wall in $\sim 30<y+<300$ to meet the recommendations for this model as suggested by ANSYS Fluent ${ }^{\circledR}$ User Manual [14]. Also, as per the studies done in [12], the $k-\varepsilon$ turbulence model performs considerably well for similar problems, considering the extra computational resources required for SST- $k-\omega$ and RSM models.

Isothermal analysis was carried out with water as the medium. The ratio of tangential velocity of the fluid to that of the disc was monitored at various radial locations along the disc. An area-weighted average value of $K_{\mathrm{gr}}$ is then determined as an overall value of $K_{\mathrm{gr}}$ for the entire seal. The results of the simulations were further validated (to be discussed in the following section) with experimental results from tests conducted on selected seal geometries.

\subsection{Experimental studies}

2.2a Test article configuration and design: A test article in which centrifugal seals of diameter up to $140 \mathrm{~mm}$ can be tested and can accommodate the variations in axial and radial gaps was configured, designed and fabricated inhouse. A close-up view of the test article is given in figure 5. The assembly consists of a seal casing (1) inside which the seal with radial slots (2) is placed for testing. A transparent enclosure made of Perspex material (3) is installed to allow visual access. Circular graduations are provided on the Perspex window to measure the radius of interface formed during seal operation. Water enters the test article through ports (4) and leaves through (5). Till the seal 


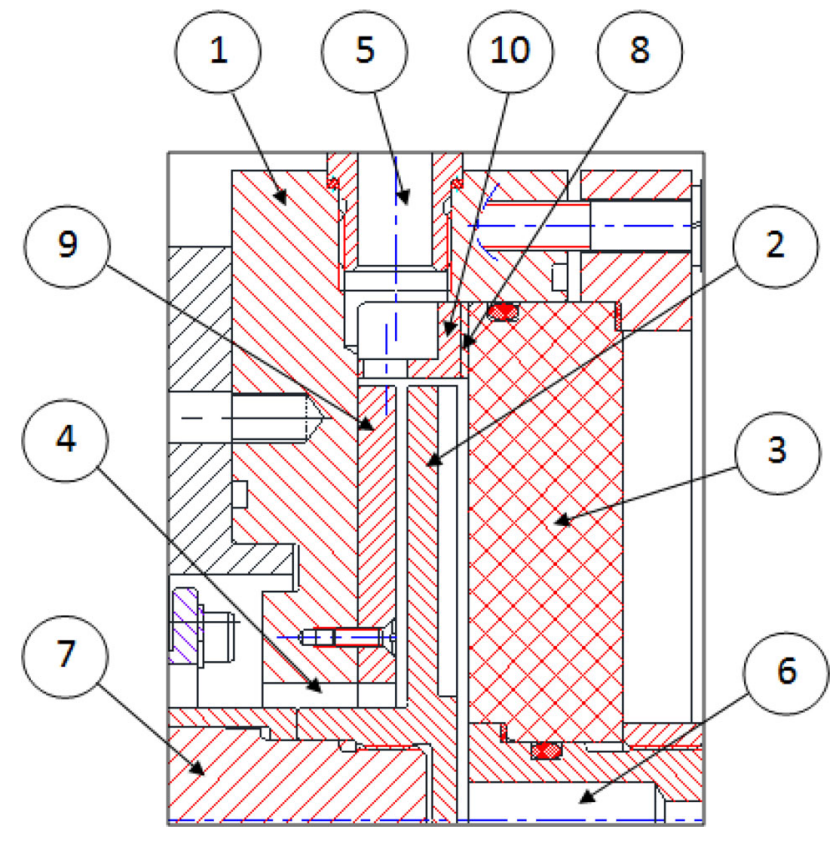

Figure 5. Detailed view of centrifugal seal test article. 1. Seal casing, 2. sealing impeller (seal), 3. Perspex window, 4. water inlet, 5. water outlet, 6 . water drain, 7. drive shaft, 8. spacer for varying seal side clearance $s_{\mathrm{gr}}$, 9. spacer for varying backside clearance $s_{\mathrm{sm}}$ and 10 . spacer for varying radial clearance ' $c$ ' as well as impeller OD ' $a$ '

becomes fully functional, a small leak is expected and is drained through (6). The seal is fixed to and driven by a shaft (7), which is connected to the torque meter and then to the drive motor through a coupling. Adequate sealing provisions are ensured to prevent leaks for various static and dynamic elements of the test article. Ring spacers (8) are used to change the axial clearance on the sealing side. Ring spacers (9) are used to change the axial clearance in the back side. Ring spacers (10) are used to change radial clearance as well as for testing seals of different diameters. A photograph of the assembled test article is given in figure 6 .

2.2b Test cell and instrumentation: A centrifugal pump with VFD drive was used as water source. A motor drive with gearbox was used to drive the seal. Water flow rate was measured by a flow meter (having inaccuracy of $\pm 0.5 \%$ ). A total of nine calibrated pressure transducers (having inaccuracy of $\pm 0.5 \%$ of the full scale reading) were used for pressure measurements at various radial locations on either side of the seal. Calibrated thermocouples (having inaccuracy of $\pm 0.5 \mathrm{~K}$ ) were used for temperature measurements at test article inlet/outlets. A high speed camera (Phantom V1210) is used to capture the liquid-air interface formed on the sealing side during operation. Videos were typically acquired using $368 \times 256$ pixels at 26,000 frames per second with the image exposure time set to $37 \mu \mathrm{s}$,

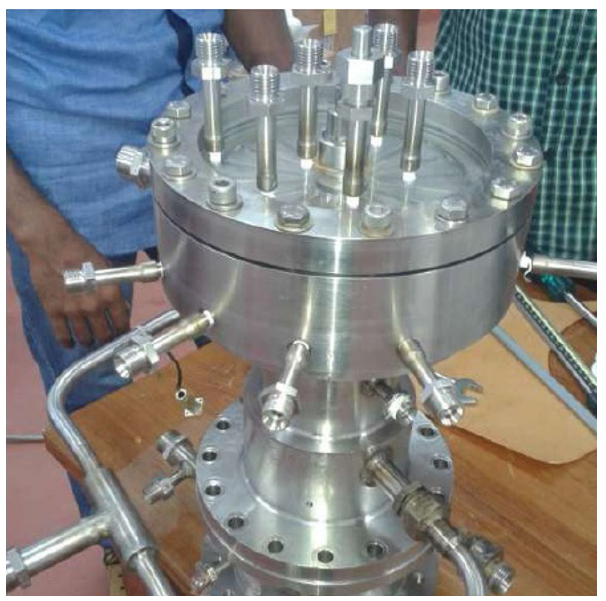

Figure 6. Centrifugal seal test article assembly.

which ensures a blur-free imaging. Lighting was provided by two $1000 \mathrm{~W}$ halogen lights placed in close proximity to the test article. The camera and light sources are properly aligned and positioned in front of the Perspex window for accurate determination of radius of the liquid-air interface. Graduations were provided on the Perspex window to enable measurement of radius of the interface from the high speed image/video with inaccuracy of $\pm 0.5 \mathrm{~mm}$ ). A calibrated torque meter (having inaccuracy of $\pm 0.5 \%$ ) was employed for torque measurements. An optical speed sensor (having inaccuracy of $\pm 0.5 \%$ ) was used for measurement of angular velocity. An uncertainty analysis was carried out as per [15] to determine the overall uncertainty in $K_{\mathrm{gr}}$ as $1-5 \%$ and moment coefficient $C_{\mathrm{m}}$ as $1.2 \%$.

2.2c Types of seal configurations studied: Photographs of the three variants of the seals tested under the current study are given in figure 7. Focus of the current study is on seal with slots (Type-1). Type-2 seals have vanes/ribs instead of slots and are more popular. Type- 3 seals are improved version of Type- 1 seals with a circular lip added over the seal outer diameter.

Overall $K_{\mathrm{gr}}$ for the sealing side is calculated from the relation

$$
K_{\mathrm{gr}}=\frac{1}{\omega} \sqrt{\frac{2\left(p_{\mathrm{tip}}-p_{\mathrm{i}}\right)}{\rho\left(a^{2}-R_{\mathrm{i}}^{2}\right)}} .
$$

The moment coefficient for the seal is calculated from the torque measurements using the relation

$$
C_{\mathrm{m}}=\frac{M}{\frac{1}{2} \rho \omega^{2} a^{5}} .
$$

The radius of interface $R_{\mathrm{i}}$ is measured from a high speed video, with the help of graduations provided on the Perspex window. A typical case is shown in figure 8 . 

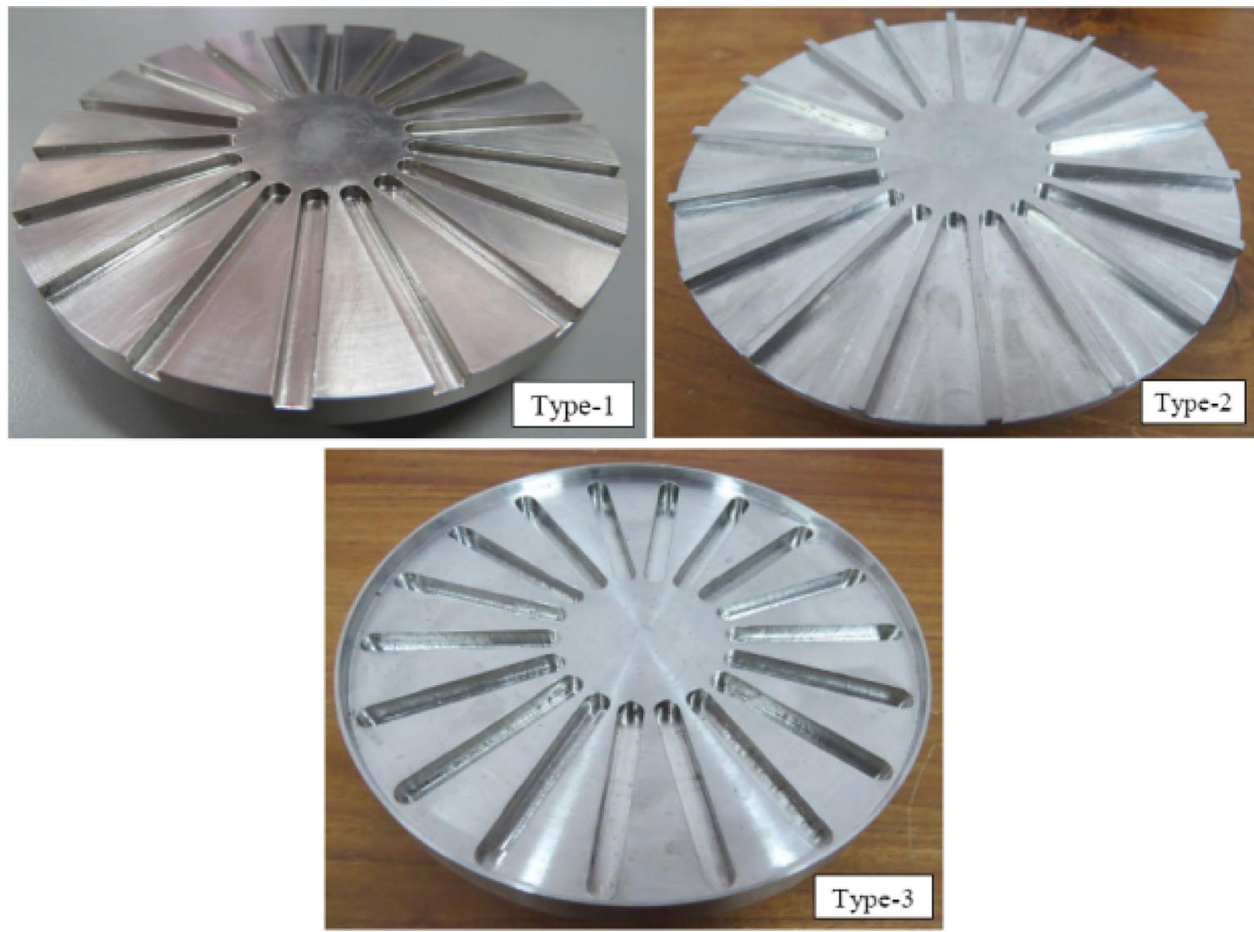

Figure 7. Types of seal configurations studied.

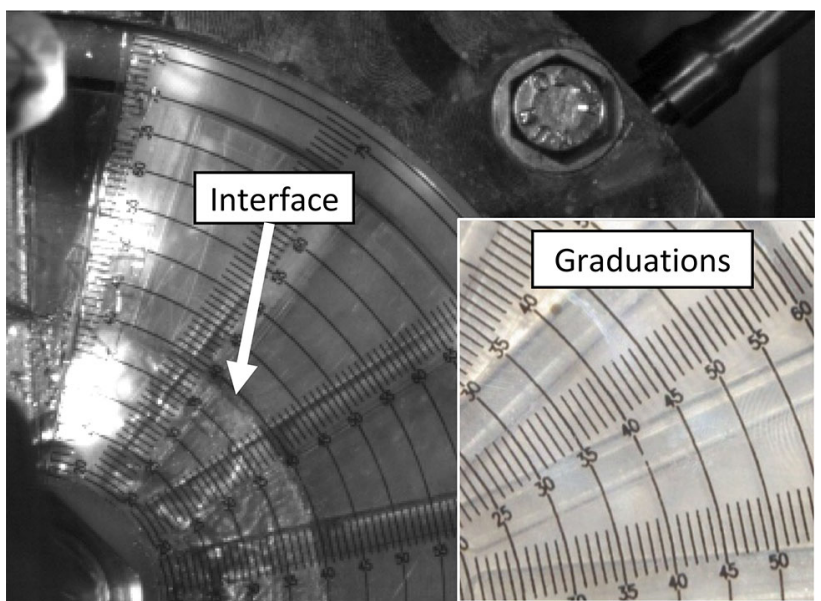

Figure 8. High speed image with $R_{\mathrm{i}}=39 \mathrm{~mm}$.

\section{Results and discussion}

\subsection{Numerical studies (sealing side geometry)}

3.1a Effect of width of slots, w: The effect of varying the width of slots is shown in figure 9. Three cases were analysed with 1,3 and $5 \mathrm{~mm}$ wide slots. $K_{\mathrm{gr}}$ increases with increasing width of slots. The improvement in $K_{\mathrm{gr}}$ is very small beyond $3 \mathrm{~mm}(\sim 3 \%)$, indicating that optimum width of slots is $3-5 \mathrm{~mm}$. The experimental value of $K_{\mathrm{gr}}$ for a disc

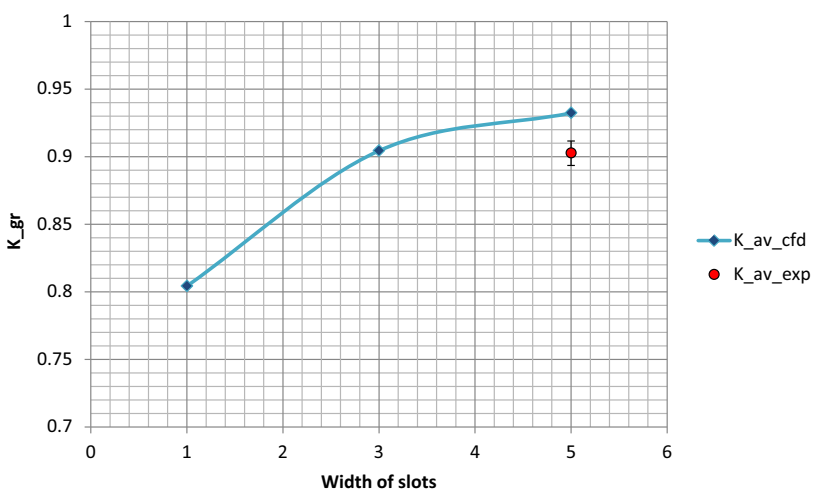

Figure 9. Effect of width of slots, $w$.

with $5 \mathrm{~mm}$ wide slots is also plotted for comparison. As the width of slots increases, the 'grip' of the disc on the fluid increases, resulting in the enhancement of tangential velocity. However, beyond some value, the added advantage of higher width does not payoff. Higher values of width of slots are countered by the difficulties involved in the fabrication process, especially for lower radii areas of the seal.

3.1b Effect of number of slots, $n$ : The effect of varying the number of slots is shown in figure 10. Four cases were analysed with $6,12,18$ and 24 slots. The improvement in $K_{\mathrm{gr}}$ is very small $(\sim 2 \%)$ beyond 18 , indicating that 


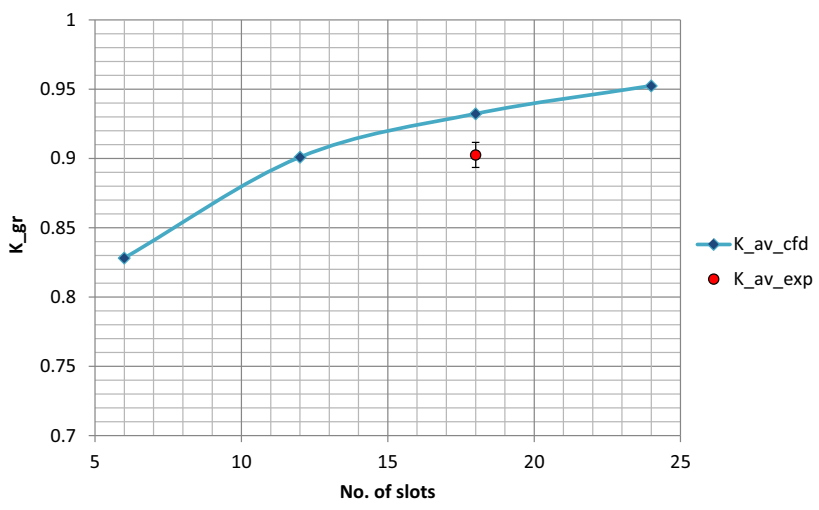

Figure 10. Effect of number of slots, $n$.

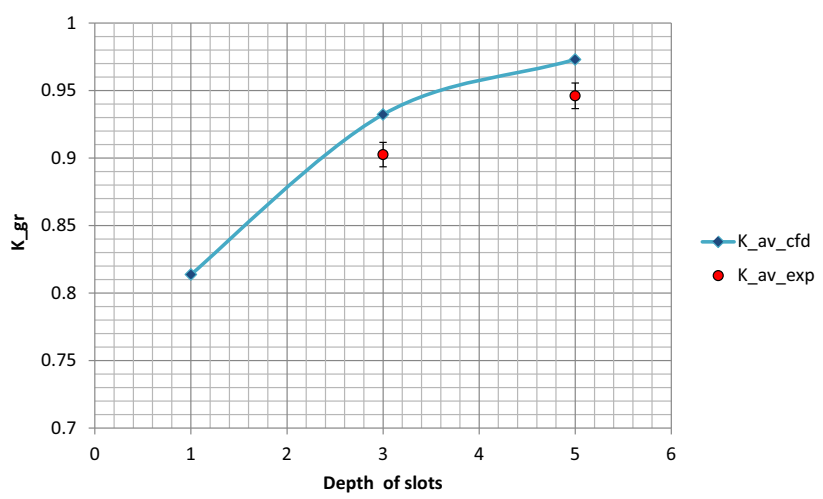

Figure 11. Effect of depth of slots, $h$.

optimum number of slots is $18-24$. As the number of slots increases, the advantage of adding more slots is countered by the difficulties involved in the fabrication process and additional cost impact.

3.1c Effect of depth of slots, $h$ : The effect of varying the depth of slots is shown in figure 11. Three cases were analysed with 1,3 and $5 \mathrm{~mm}$ deep slots. Since the improvement in $K_{\mathrm{gr}}$ is very small beyond $3 \mathrm{~mm}(\sim 4 \%)$, the recommended range for depth of slots is $3-5 \mathrm{~mm}$. Higher depth of slots demands larger thickness of the disc and hence has a weight penalty.

The marginal difference between the numerical and experimental results (maximum difference only $\sim 3.2 \%$, considering all such cases shown in figures 9-11) is expected to be a result of the inferior performance of the $k-$ $\varepsilon$ turbulence model with wall function approach when compared with SST- $k-\omega$ and RSM turbulence models.

\subsection{Performance tests on seals}

3.2a Influence of radius of interface, $\bar{R}_{\mathrm{i}}$ : The sealing capacity as a function of the non-dimensional radius of

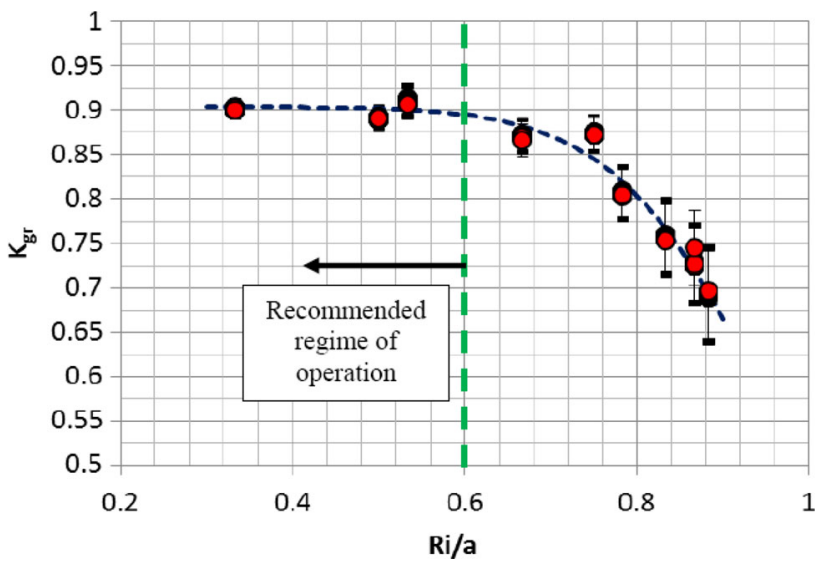

Figure 12. Dependence of $K_{\mathrm{gr}}$ on $\bar{R}_{\mathrm{i}}$ for Type-1 seal.

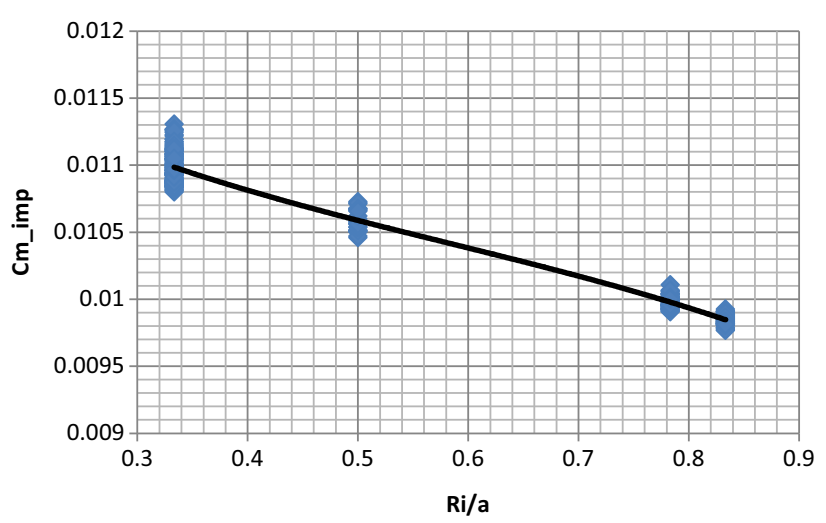

Figure 13. Dependence of $C_{\mathrm{m}}$ on $\bar{R}_{\mathrm{i}}$ for Type-1 seal.

interface $\bar{R}_{\mathrm{i}}=R_{\mathrm{i}} / a$ is plotted in figure 12. $K_{\mathrm{gr}}$ drops beyond $\bar{R}_{\mathrm{i}}>0.6$ due to the retarding effect of the cylindrical wall above the rotor. A similar effect was observed by Ketola and McGrew [6] in their studies on plain discs. The moment coefficient also showed a decreasing trend with respect to increasing $\bar{R}_{\mathrm{i}}$ (see figure 13), which is due to the reduction in wetted area of the disc.

$3.2 \mathrm{~b}$ Influence of sealing side axial clearance, $K_{\mathrm{gr}}$ : The nature of variation of $K_{\mathrm{gr}}$ and $C_{\mathrm{m}}$ with respect to changes in the axial clearance on the sealing side expressed in nondimensional form as $(s / a)_{\mathrm{gr}}$ is shown in figure 14. The sealing capacity seems to be unaffected in the range considered; however, the moment coefficient shows a significant increase $(\sim 40 \%)$ as $(s / a)_{\mathrm{gr}}$ is increased. The increase in $C_{\mathrm{m}}$ is attributed to the increase in the volume of fluid swirled by the disc. However, too low clearances demand for precise manufacturing and assembly requirements. Also, according to [5] at very low values of $(s / a)$, the flow regime shifts to regime III (figure 2), wherein $C_{\mathrm{m}}$ shows increasing trend with respect to further reduction in axial 


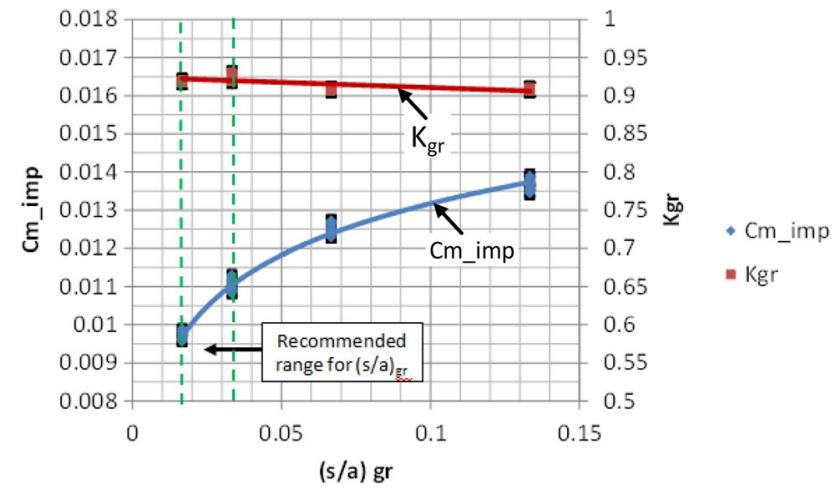

Figure 14. Dependence of $K_{\mathrm{gr}}$ and $C_{\mathrm{m}}$ on $(s / a)_{\mathrm{gr}}$.

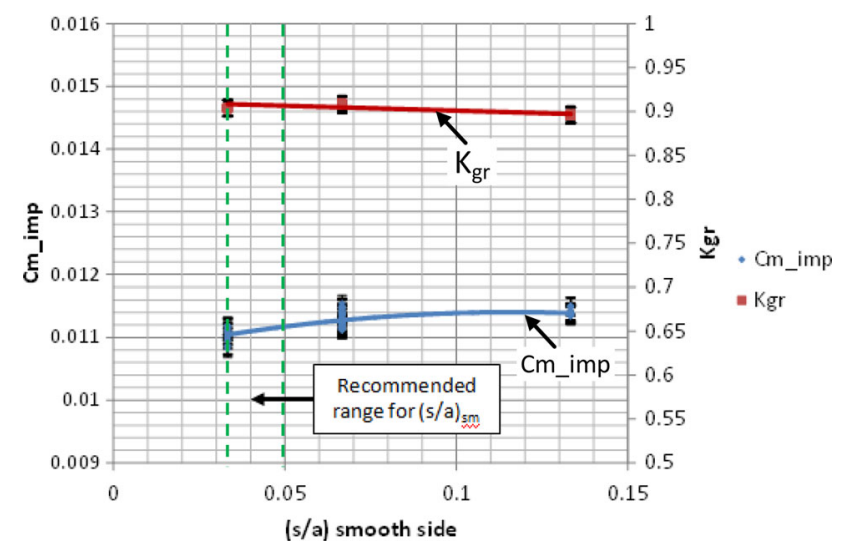

Figure 15. Dependence of $K_{\mathrm{gr}}$ and $C_{\mathrm{m}}$ on $(s / a)_{\mathrm{am}}$

clearance. Considering all these aspects, a value of clearance $s_{\mathrm{gr}}=1-2 \mathrm{~mm}$ is recommended.

3.2c Influence of back side axial clearance, $K_{\mathrm{sm}}$ : Sealing capacity is unaffected as in the previous case (see figure 15). There is no drastic change in moment coefficient; however, a marginal reduction in moment coefficient $(\sim 3.5 \%)$ is seen as $(s / a)_{\mathrm{sm}}$ is reduced. Since the moment due to the sealing side is much higher than that on the back side, the change in the moment coefficient of the total seal is very small. As mentioned earlier, very low clearances demand for precise manufacturing and assembly requirements. Considering this, the recommended values for the back side gap can be taken as $2-3 \mathrm{~mm}$.

$3.2 \mathrm{~d}$ Influence of radial clearance, $c$ : Sealing capacity is unaffected as in the previous case (see figure 16). However, there is a considerable reduction in moment coefficient $(\sim 15 \%)$ as $(c / a)$ is reduced. Lower radial clearances have minimum power consumption, but demand more precision manufacturing and assembly techniques. Hence a clearance of $0.5-1 \mathrm{~mm}$ seems to be the best choice for this case.

3.2e Influence of throughflow rate $Q$ : The effect of throughflow is to suppress the radial pressure gradient

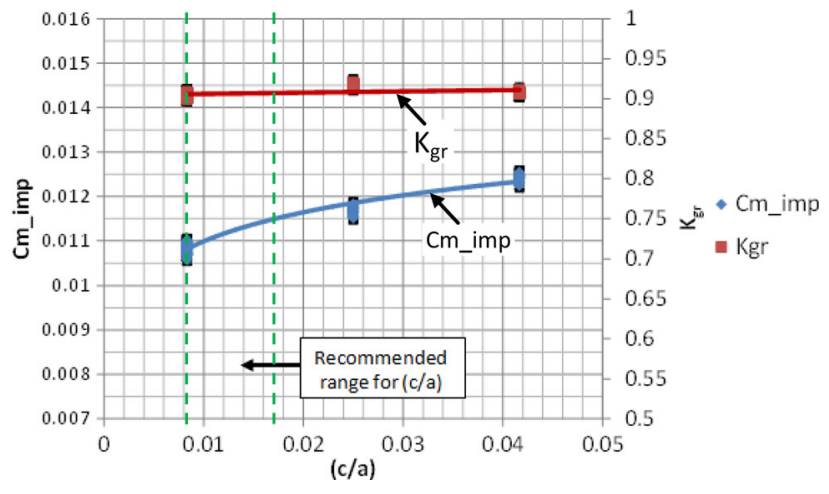

Figure 16. Dependence of $K_{\mathrm{gr}}$ and $C_{\mathrm{m}}$ on $c / a$.

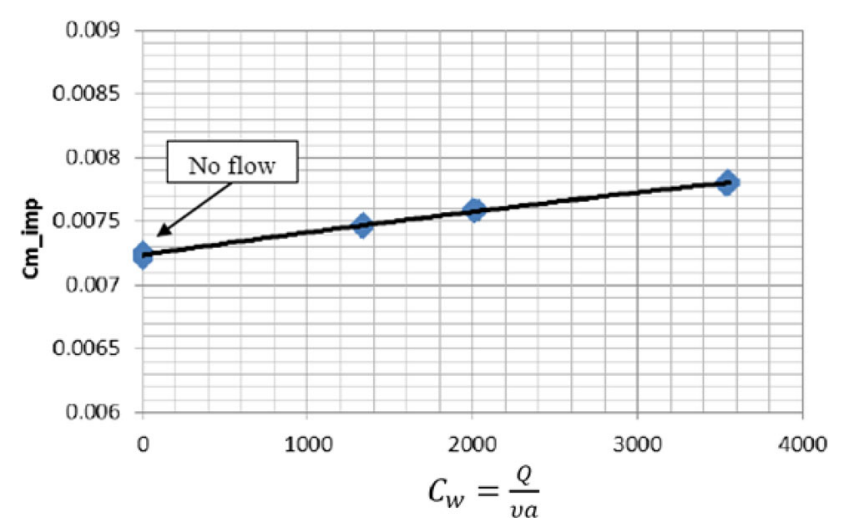

Figure 17. Dependence of $C_{\mathrm{m}}$ on $C_{\mathrm{w}}$.

along the back side of the seal and increase the power consumption $(\sim 8 \%)$. The reduction in radial pressure gradient modifies $\bar{R}_{\mathrm{i}}$, whose influence on $K_{\mathrm{gr}}$ has already been presented. The influence on $C_{\mathrm{m}}$ with respect to flow rate expressed in non-dimensional form as $C_{\mathrm{w}}$ is presented in figure 17. It is inferred that for optimum performance, minimum required flow rate to satisfy the constraints on the temperature rise of the operating fluid alone needs to be provided.

\subsection{Performance tests on seals}

3.3a Comparison with Type-2 seal performance: The sealing capacity and moment coefficients for Type- 1 and Type -2 seals are compared, respectively, in figures 18 and 19. Geometrical and operational parameters for Type- 2 are selected to match with those of Type-1.

There is a marginal improvement in the sealing capacity ( $\sim 6 \%)$ of Type- 2 seals when compared with Type-1, however at the cost of a much higher increase in $C_{\mathrm{m}}$ ( $\sim 20 \%$ ). The higher volume of fluid entrapped within the vanes of Type- 2 accounts for the higher torque. This indicates a scope for an improved configuration of Type- 1 seal. 


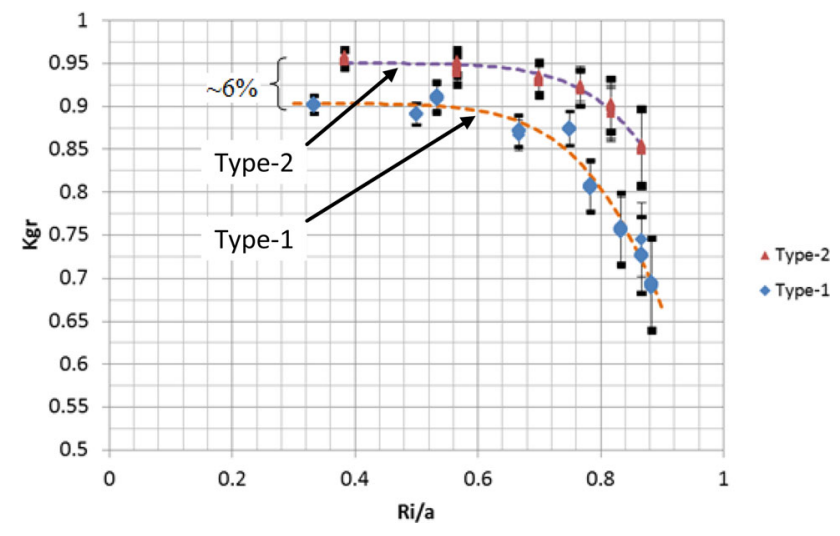

Figure 18. $K_{\mathrm{gr}}$ comparison - Type-1 and -2 seals.

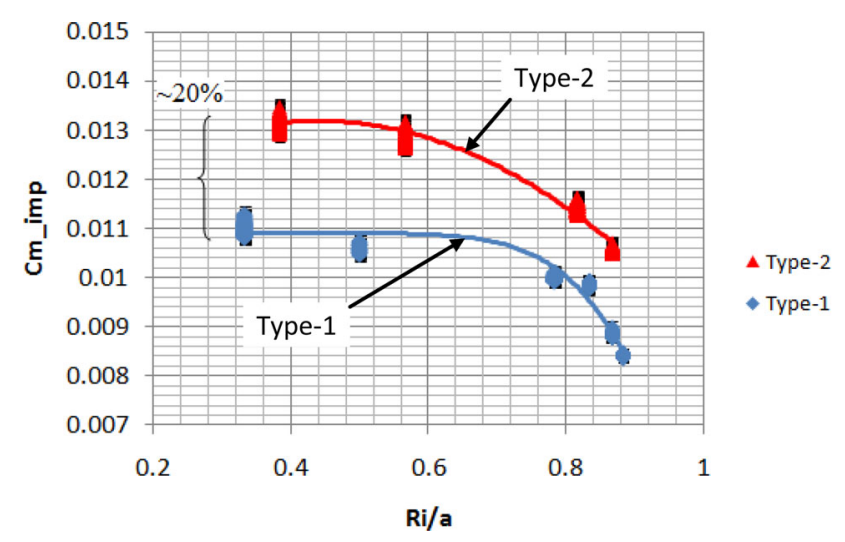

Figure 19. $C_{\mathrm{m}}$ comparison - Type-1 and -2 seals.

3.3b Improved seal configuration (Type-3): To improve the sealing capacity of Type-1 seals a circumferential lip was added to the existing design to reduce the influence of stationary shroud. Also the depth of groves was increased from 3 to $5 \mathrm{~mm}$. Out of the three possible assembly configurations, the optimum configuration of the resulting seal (Type-3(C)) is shown in figure 20.

Type-3(C) seal has sealing capacity equal to that of the conventional one (Type-2); at the same time it has a lower value of $C_{-\mathrm{m}}(11-14 \%)$. A comparison of the same is given in figures 21 and 22 . The drop in sealing capacity of the Type- 3 seal occurs only beyond $\bar{R}_{\mathrm{i}}>0.8$ in place of 0.6 for the Type- 1 seals.

\subsection{Simple 1D model for seal operation}

Since sealing side is experimentally characterized from our tests and established empirical correlations are available for the back side of the seal from [11], it would be advantageous to have a simple model that could predict the location of interface for a given set of geometrical and operational parameters. The model is formulated based on the following inputs:

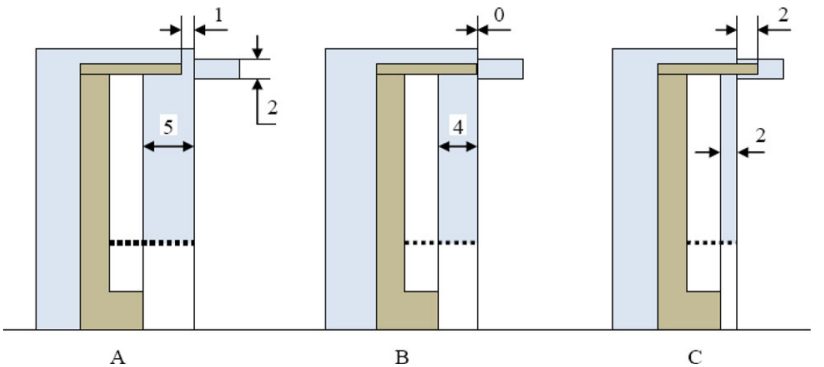

Figure 20. Assembly configurations for Type-3 seals.

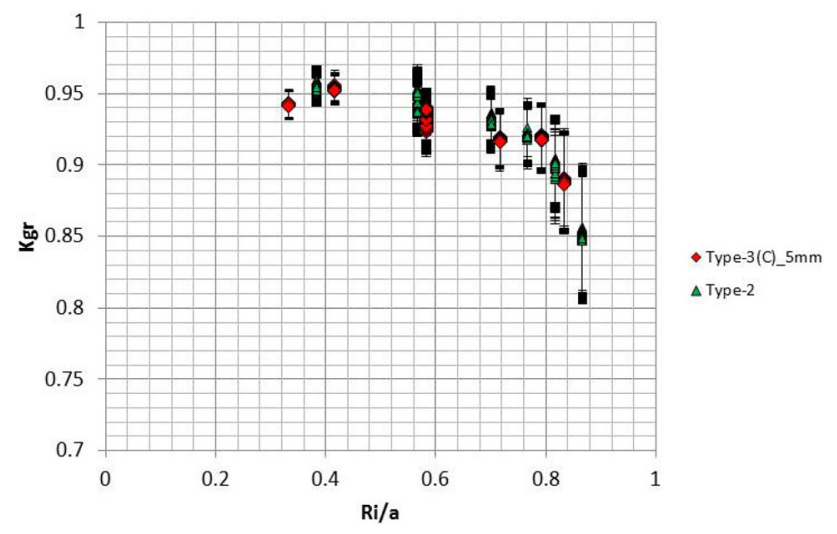

Figure 21. $K_{\mathrm{gr}}$ comparison - Type-2 and -3 seals.

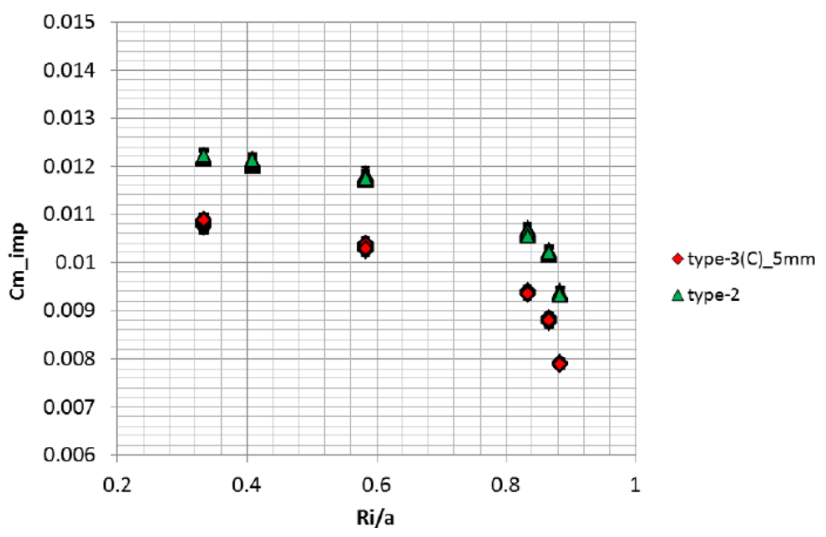

Figure 22. $C_{\mathrm{m}}$ comparison - Type- 2 and -3 seals.

- applied pressure difference across seal,

- radius of the disc,

- angular velocity of shaft,

- throughflow rate (for back side) and

- properties of working medium.

A schematic of the model domain showing the various model parameters is given in figure 23.

3.4a Modelling back side of seal: To model the back side of the seal, correlations for $K$ as $f\left(\mathrm{Cq}_{\mathrm{r}}\right)$ provided by Poncet in 


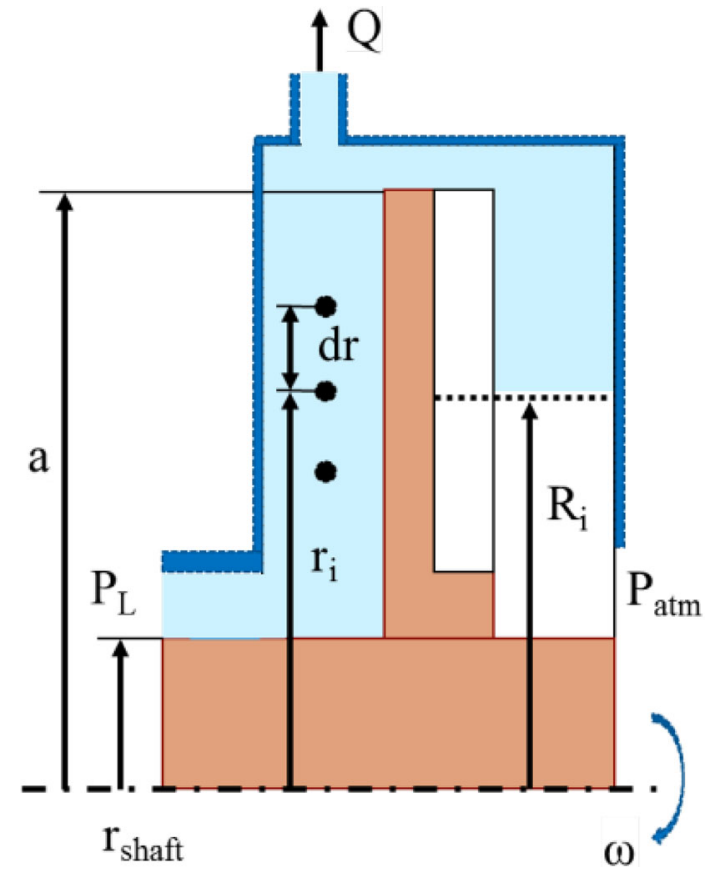

Figure 23. Model domain and model parameters.

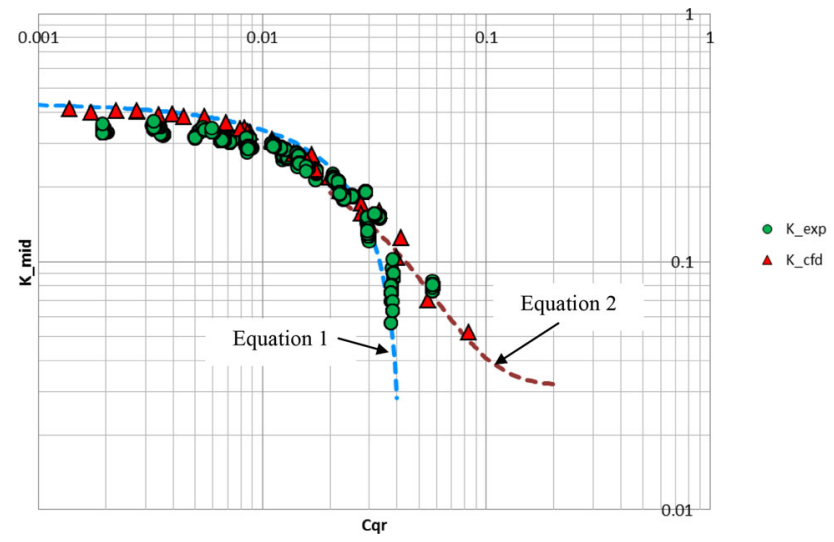

Figure 24. $K$ vs. $\mathrm{Cq}_{\mathrm{r}}$ from our tests with plain disc and CFD simulations compared to correlations given by Poncet [11].

[11] were validated through tests on the plain disc of the same diameter as that of the seal using the same seal test facility and implemented to calculate the pressure rise during seal operation. The $K$ values were estimated from radial pressure measurements while the throughflow rate and speed were varied during a particular test. The variation of $K$ with respect to $\mathrm{Cq}_{\mathrm{r}}$ obtained from our tests and CFD simulations is compared with the correlations provided by Poncet in [11] in figure 24. A very good match has been obtained as seen from the figure. At low values of $\mathrm{Cq}_{\mathrm{r}}$, the flow structure consisting of a rotating inviscid core surrounded by two boundary layers each on the stator and rotor exists. $\mathrm{As} \mathrm{Cq}_{\mathrm{r}}$ increases, $K$ starts dropping. At very large
Table 2. Empirical correlations used for $K \mathrm{vs} . \mathrm{Cq}_{\mathrm{r}}$ for back side of seal.

\begin{tabular}{ccc}
\hline No. & Equation & Range \\
\hline 1 & $K_{\text {core }}=2 \times\left(-5.9 \mathrm{Cq}_{\mathrm{r}}+0.63\right)^{\frac{5}{7}}-1$ & $\mathrm{Cq}_{\mathrm{r}} \leq 0.02$ \\
2 & $K_{\text {no_core }}=0.032+0.32 e^{-\frac{\mathrm{Cq}_{\mathrm{r}}}{0.028}}$ & $\mathrm{Cq}_{\mathrm{r}} \geq 0.04$ \\
3 & $K_{\text {average }}=\frac{K_{\text {core }}+K_{\text {no_core }}}{2}$ & $0.02<\mathrm{Cq}_{\mathrm{r}}<0.04$ \\
\hline
\end{tabular}

flow rates, the core is completely suppressed by the large throughflow rate. The correlations used for various ranges of $\mathrm{Cq}_{\mathrm{r}}$ is given in table 2 .

The back side of the seal was split into several very small radial segments and appropriate correlations were applied selectively from table 2 to each segment depending on the local value of $\mathrm{Cq}_{\mathrm{r}}$ to determine $K$ and pressure rise for that small segment. Finally, summation of individual pressure rises gives the total pressure rise across the whole disc. The radial pressure rise across $i$ th radial segment is calculated using the relation

$$
p_{i+1}=p_{i}+\frac{1}{2} \rho(K \omega)^{2}\left(r_{i+1}^{2}-r_{i}^{2}\right)
$$

3.4b Modelling vaned/grooved side of seal: The radius of interface $R_{\mathrm{i}}$ is then calculated based on an initial guess for $K_{\mathrm{gr}}$ and further updated based on the dependence of $K_{\mathrm{gr}}$ on $R_{\mathrm{i}}$ for Type-1 seal (figure 12) given by the expression

$$
K_{\mathrm{gr}}=\left(1.107+1.029 \bar{R}_{\mathrm{i}}^{9.062}\right)^{-1} .
$$

$R_{\mathrm{i}}$ is recalculated with the new $K_{\mathrm{gr}}$ using (2) and this process is repeated till difference in the calculated $R_{\mathrm{i}}<10^{-3}$. This model was implemented in Matlab ${ }^{\circledR}$ and a very good match was obtained for the $R_{\mathrm{i}}$ with respect to experimental results. The model can be extended to any type of seal by suitably updating the performance characteristic equation (5).

The same model can also be used as a quick centrifugal seal design tool for a given pressure difference across the seal, shaft speed, throughflow rate and working medium wherein the required seal diameter can be estimated iteratively, starting from an initial guess.

\section{Conclusions}

Performance of centrifugal seals with slots provided on the sealing side (Type-1) has been characterized. The effect of various geometrical and operating parameters on the sealing capacity and power consumption was studied experimentally and numerically. An optimum geometrical configuration for the sealing side of the centrifugal seal (Type-1) was arrived at in terms of sealing capacity. An improved version (Type-3) seal was found to have superior performance over the conventional seal (Type-2). The 
limiting value of $\bar{R}_{\mathrm{i}}$ for initial seal configuration was also increased from $60 \%$ to $80 \%$ by this modification. A simple 1D model has been developed to predict the radius of interface during seal operation, which also can be used as a quick design tool for centrifugal seals.

\section{Acknowledgement}

The authors would like to acknowledge the efforts of the team at Turbopump Lab, LPSC, Valiamala, towards the tests done on seals, employees of Proto Fabrication Facility (PFF) at LPSC, Valiamala, and Manufacturing Lab, IIST, for the realization of components required and Thermal Lab, IIST, for arrangements made for the experimental setup. The authors also would like to thank Dr Prathap C, IIST, Valiamala, for providing the high speed camera.

\section{Nomenclature}

$a \quad$ outer radius of disc/seal

$c$ radial clearance between disc/seal and housing

$C_{\mathrm{w}} \quad$ flow rate coefficient, $C_{\mathrm{w}}=\frac{Q}{v a}$

$\mathrm{Cq}_{\mathrm{r}} \quad$ throughflow rate coefficient, $\mathrm{Cq}_{\mathrm{r}}=\frac{Q\left(\frac{\omega r^{2}}{v}\right)^{\frac{1}{5}}}{2 \pi r^{3} \omega}$

$C_{\mathrm{m}} \quad$ moment coefficient

$h$ depth of slots/height of vanes on seal geometry

$K$ core rotation factor

$R_{\mathrm{i}} \quad$ radius of interface

$n \quad$ number of slots/vanes

$p$ pressure

$Q \quad$ superposed throughflow rate

$\bar{R}_{\mathrm{i}} \quad$ non-dimensional radius of interface defined as $\bar{R}_{\mathrm{i}}=\frac{R_{i}}{a}$

Re Reynolds number, $R e=\frac{\omega a^{2}}{v}$

$s \quad$ axial clearance between seal and housing

$\omega$ angular velocity of disc/seal

$v \quad$ kinematic viscosity of operating medium

$w \quad$ width of slot/vane

\section{Subscripts}

_sm smooth side of seal

_gr sealing side of seal (with grooves/vanes)

_imp parameter for the whole seal/impeller/rotor _exp_parameter estimated from experimental data under current work

_emp parameter estimated from empirical expressions

_CFD parameter estimated from CFD results

\section{References}

[1] Heinz K Muller and Bernaud S Nau 1998 Fluid sealing technology: principles and applications. New York: Dekker

[2] Theodor Von Karman 1921 On laminar and turbulent friction. NASA Technical Memorandum No. 1092

[3] Cochran W G 1934 The flow due to a rotating disc. Math. Proc. Cambridge Philos. Soc. 30: 365-375

[4] Goldstein S 1935 On the resistance to the rotation of a disc immersed in a fluid. Proc. Cambridge Philos. Soc. 31: 232-241

[5] Daily J W and Nece R E 1960 Chamber dimension effects on induced flow and frictional resistance of enclosed rotating disks. J. Basic Eng. 82: 217-232

[6] Ketola H N and McGrew J M 1968 Pressure, frictional resistance and flow characteristics of the partially wetted rotating disk. J. Lubr. Technol. 90(2): 395-404

[7] Owen J M 1989 An approximate solution for the flow between a rotating and a stationary disk. J. Turbomach. 111: 323-332

[8] Arndt R E A 1960 Effect of superposed throughflows on motion induced by enclosed rotating discs. New York: City College of New York

[9] Due H F 1966 An empirical method for calculating radial pressure distribution on rotating disks. J. Eng. Power 88(2): 188-196

[10] Marcu B, McGlynn R D and Ferguson T V 2008 On the performance of dynamic shaft seals used in liquid propellant rocket turbopumps. In: Proceedings of the 44th AIAA/ASME/ SAE/ASEE Joint Propulsion Conference and Exhibit, pp. $1-16$

[11] Poncet S 2005 Centrifugal flow in a rotor-stator cavity. $J$. Fluids Eng. 127: 787-794

[12] Poncet S, Soghe R Da and Facchini B 2010 RANS modeling of flow in rotating cavity system. In: Proceedings of the European Conference on Computational Fluid Dynamics, Lisbon, Portugal, pp. 1-20

[13] Beretta G P and Malfa E 2003 Flow and heat transfer in cavities between rotor and stator disks. Int. J. Heat Mass Transfer 46: 2715-2726

[14] ANSYS Fluent theory guide R14.5

[15] Bevington P R and Keith Robinson D 2003 Data reduction and error analysis for the physical sciences, 3rd ed. Boston: McGraw-Hill 